\title{
Accuracy of 3 Tesla pelvic phased-array multiparametric MRI in diagnosing prostate cancer at repeat biopsy
}

\author{
Pietro Pepe ${ }^{1}$, Antonio Garufi ${ }^{2}$, Giandomenico Priolo ${ }^{2}$, Giuseppe Dibenedetto ${ }^{1}$, Michele Salemi ${ }^{4}$, \\ Michele Pennisi ${ }^{1}$, Filippo Fraggetta ${ }^{3}$, Francesco Aragona ${ }^{1}$, Michele Barbera ${ }^{5}$ \\ ${ }^{1}$ Urology Unit, ${ }^{2}$ Imaging Department and ${ }^{3}$ Pathology Unit, Cannizzaro Hospital, Catania, Italy; \\ ${ }^{4}$ Section of Endocrinology, Andrology and Internal Medicine, Department of Medical and Pediatric Sciences, \\ University of Catania, Italy; \\ ${ }^{5}$ Urology Unit, Sciacca, Italy.
}

\begin{abstract}
Summary Introduction. Multiparametric pelvic magnetic resonance imaging (mpMRI)
\end{abstract} accuracy in prostate cancer $(\mathrm{PCa}$ ) diagnosis was evaluated. Materials and Methods. From June 2011 to December 2013, 168 patients (median 65 years) with negative digital rectal examination underwent repeat transperineal saturation biopsy (SPBx; median 28 cores) for persistently high or increasing PSA values, PSA $>10 \mathrm{ng} / \mathrm{ml}$ or PSA values between 4.1-10 or 2.6-4 $\mathrm{ng} / \mathrm{ml}$ with free/total PSA $<25 \%$ and $<20 \%$, respectively. All patients underwent mpMRI using a 3.0 Tesla scanner equipped with surface 16 channels phased-array coil and lesions suspicious for PCa were submitted to additional targeted biopsies.

Results. A T1c PCa was found in 66 (39\%) cases; SPBx and mpMRI-suspicious targeted biopsy diagnosed 60 (91\%) and $52(78.8 \%)$ cancers missing 6 (all of the anterior zone) and 14 cancers (12 and 2 of the lateral margins and anterior zone), respectively; in detail, mpMRI missed 12 (18.1\%) PCa charaterized by microfocal (1 positive core with greatest percentage of cancer and Gleason score equal to 5\% and 6, respectively) disease at risk for insignificant cancer. The diameter of the suspicious mpMRI lesion was directly correlated to the diagnosis of $\mathrm{PCa}$ with poor Gleason score $(p<0.05)$; detection rate of cancer for each suspicious mpMRI core was 35.3\%. Diagnostic accuracy, sensitivity, specificity, positive and negative predictive value of mpMRI in diagnosing PCa was $75.7 \%, 82.5 \%$, $71.8 \%, 78.9 \%, 87.9 \%$, respectively.

Conclusion. Multiparametric pMRI improved SPBx accuracy in diagnosing significant anterior PCa; the diameter of mpMRI suspicious lesion resulted significantly predictive of aggressive cancers.

KEY WORDS: Prostate cancer; Multiparametric MRI; Prostate targeted biopsy; Pelvic phased-array MRI..

Submitted 12 January 2014; Accepted 31 October 2014

\section{INTRODUCTION}

Although extended (12-18 cores) and saturation biopsy (SPBx; > 20 cores) have been suggested $(1,2)$ to improve detection rate for prostate cancer $(\mathrm{PCa})$, repeat prostate biopsy, still today, constitutes about 30\% of the entire procedure with an estimated diagnosis of cancer equal to $20-40 \%$. Therefore, the ideal biopsy scheme should perform targeted biopsies to diagnose only significant $\mathrm{PCa}$, reducing the number of unnecessary procedures and false negative rate. In this light, multiparametric magnetic resonance imaging (mMRI) using pelvic phased-array coil (mpMRI) or endorectal coil (meMRI) has been proposed as a more accurate alternative in comparison with transrectal ultrasound (TRUS) to increase the detection rate for PCa, especially in case of repeat biopsy (3-16).

The accuracy of mpMRI in diagnosing PCa in men submitted to repeat biopsy was prospectively evaluated.

\section{MateriaLs AND METHOdS}

From June 2011 to December 2013, 168 patients, all of Caucasian origin and between the ages of 49 and 75 years (median 65 years), with negative digital rectal examination underwent SPBx (median 28, range: 6-35 cores) for persistent suspicion of PCa. The 168 patients enrolled in a prospective, monocentric and multi-departmental study were selected from a case-finding protocol for PCa detection (17) and had one single previous negative extended transperineal biopsy (18 cores) performed at least six months before (range: 6-20 months); the indications for repeat SPBx were: persistently high or increasing PSA value, PSA $>10 \mathrm{ng} / \mathrm{ml}$ or PSA values between $4.1-10$ or $2.6-4 \mathrm{ng} / \mathrm{ml}$ with free/total PSA $<25 \%$ and $<20 \%$, respectively.

All patients, provided a written informed consent, underwent mpMRI 3-10 days before undergoing the SPBx. All examinations were performed using a 3.0 Tesla scanner, (ACHIEVA 3T; Philips Healthcare Best, the Netherlands) equipped with surface 16 channels phased-array coil placed around the pelvic area with the patient in supine position; multiplanar turbo spin-echo T2-weighted (T2W), axial diffusion weighted imaging (DWI), axial dynamic contrast enhanced (DCE) and spectroscopy were performed for each patient (Figure 1). The criteria (14) for a positive lesion on T2W (Figure 1) were the presence of a circumscribed, low signal intensity lesion (hypointense); a posi- 
Figure 1.

3 Tesla pelvic phased-array multiparametric MRI patterns in patients with prostate cancer.
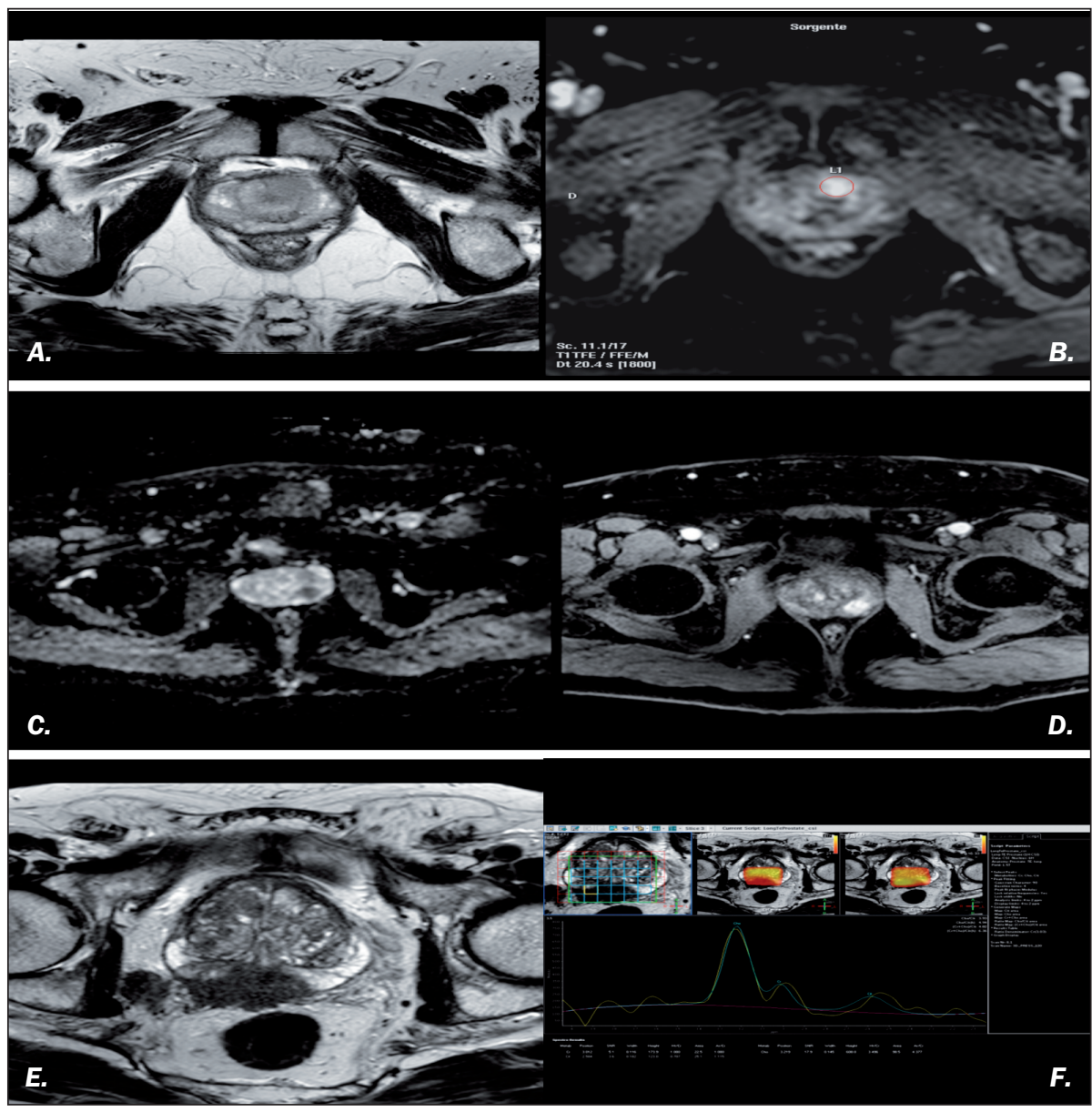

- T2-weighted (a) and DCE (b) MRI of an anterior nodular area.

- T2-weighted (c) and DCE (d) MRI of a peripheral nodular area (1 cm).

- Large (e) peripheral T2-weighted hypointense area with positive spectroscopy (f) that exceeded the surrounding tissue.

tive lesion on DCE (Figure 1) was characterized by the presence of foci showing early and intense enhancement and rapid washout after power injection $(3.0 \mathrm{ml} / \mathrm{s})$ of gadobutrol $0.1 \mathrm{ml} / \mathrm{kg}$ (Gadovist $^{\circledR}$; Bayer Schering Pharma, Germany) followed by a $15 \mathrm{ml}$ saline flush. A positive lesion on spectroscopy (Figure 1) was any area where the choline to citrato ratio was 3 or more standard deviations above the mean healthy value. Two radiologists (AF, GP) blinded to pre-imaging clinical parameters evaluated the MRI data separately and independently.

SPBx was performed transperineally using a tru-cut 18 gauge needle (Bard; Covington, GA USA) and a GE Logiq 500 PRO ecograph (General Electric; Milwaukee, WI USA) supplied with a biplanar transrectal probe $(5-6.5 \mathrm{MHz})$ under sedation and antibiotic prophylaxis (18). To ensure that histopatological findings matched with MRI images the assessment of radiological images and SPBx scheme were performed dividing the prostate into 14 regions: apex, middle zone and base of posterior zone for each lobe beginning parasagittally to reach the outer edges of the gland (six regions for each lobe), anterior and transitional zone (15). In the presence of mpMRI lesions suspicious for cancer, 3-4 (median: median 3.5 cores) targeted TRUS guidedbiopsies in addition to standard SPBx were performed. A probability (p) level of less than 0.05 was considered statistically significant.

\section{Results}

All patients had negative TRUS; median PSA was 10.4 $\mathrm{ng} / \mathrm{ml}$ (range: 3.7-45 $\mathrm{ng} / \mathrm{ml}$ ): 69 (41\%) had PSA > $10 \mathrm{ng} / \mathrm{ml}$, 92 (54.8\%) between 4-10 $\mathrm{ng} / \mathrm{ml}$ and 7 (4.2\%) between 2.6-4 ng/ml, respectively. Multiparametric pMRI was positive in $94(56 \%)$ out of 168 patients (Table 1); in the patients submitted to mpMRIsuspicious targeted biopsy, 329 cores were performed; in 84 (89.3\%) out of 94 the lesions were included in the SPBx scheme, on the contrary, in 10 $(10.7 \%)$ cases, the suspicious areas were localized in the anterior zone (Figure la, 1b) near the bladder neck. None had significant complications from SPBx that needed hospital admission; moreover, the mpMRI procedure was well tolerated and successfully performed in all cases.

A Tlc PCa was found in 66 (39\%) out of 168 patients and normal parenchyma in the remaining 102 (61\%). Clinical parameters, biopsy quantitative histology, Gleason score (GS) and mpMRI findings in the presence of PCa and normal parenchyma are listed in Table 1. SPBx scheme and mpMRI-suspicious targeted biopsy diagnosed 60 (91\%) and $52(78.8 \%)$ out of $66 \mathrm{PCa}$, respectively; in detail, SPBx and mpMRI missed 6 (all of the anterior zone) and 14 cancers (12 and 2 of the lateral margins and anterior zone) equal to $9 \%$ and $21.2 \%$ of the cases, respectively. In the presence and absence of $\mathrm{PCa}$ the nodular suspicious mpMRI lesions (Figure 1) had a median diameter equal to $12 \mathrm{~mm}($ range $=5-32 \mathrm{~mm})$ vs $6 \mathrm{~mm}($ range $=5-13 \mathrm{~mm})$, respectively; a cut-off of $10 \mathrm{~mm}$ resulted predictive of cancer in 22 out 27 patients ( $81.5 \%$ of the cases) with a false positive rate of $9.6 \%$. In detail, mpMRI missed 12 (18.1\%) PCa charaterized by microfocal biopsy disease (1 positive core with a greatest percentage of cancer and Gleason score equal to $5 \%$ and 6 , respectively) (19) at risk for insignificant $\mathrm{PCa}$ (cancer volume $<0.5 \mathrm{ml}$ and $\mathrm{GS}<6$ ) (20). Median nodular diameter on mpMRI was correlated to GS (GS $6=9 \mathrm{~mm}$; GS $7=16 \mathrm{~mm}$; GS $8=20 \mathrm{~mm}$ ) showing a significantly difference between GS 6 vs GS 8 $(p<0.05)$. The detection rate of cancer for each core performing SPBx vs mpMRI targeted biopsy was $9.5 \%$ vs 
Table 1.

Patients' characteristics.

\begin{tabular}{|c|c|c|c|c|c|}
\hline $\begin{array}{l}\text { Overall } \\
\text { No of patients: } 168\end{array}$ & $\begin{array}{c}\text { PCa } \\
66(39 \%)\end{array}$ & $\begin{array}{c}\text { GS } 6 \\
44\end{array}$ & $\begin{array}{c}\text { GS } 7 \\
18\end{array}$ & $\begin{array}{c}\text { GS } 8 \\
4\end{array}$ & $\begin{array}{c}\text { Normal parenchyma } \\
102(61 \%)\end{array}$ \\
\hline Median PSA ng/ml (range: 3.7-45) & 10.6 & 12 & 10 & 16 & 10.3 \\
\hline Positive mpMRI & $52(78.8 \%)$ & $30(68.2 \%)$ & $18(100 \%)$ & $4(100 \%)$ & $40(39.2 \%)$ \\
\hline Positive T2W (hypointense area) & $52(78.8 \%)$ & $23(52.2 \%)$ & $18(100 \%)$ & $4(100 \%)$ & $40(39.2 \%)$ \\
\hline Positive DWI & $51(77.3 \%)$ & $25(56.8 \%)$ & $18(100 \%)$ & $4(100 \%)$ & $37(36.2 \%)$ \\
\hline Positive DCE & $52(78.8 \%)$ & $25(56.8 \%)$ & $18(100 \%)$ & $4(100 \%)$ & $36(35.3 \%)$ \\
\hline Positive spectroscopy & $29(43.4 \%)$ & $2(12.5 \%)$ & $14(77.8 \%)$ & $4(100 \%)$ & $36(35.3 \%)$ \\
\hline Negative mpMRI & $14(21.2 \%)$ & $14(31.8 \%)$ & 0 & 0 & $62(60.8 \%)$ \\
\hline GPC & $40 \%(2-100 \%)$ & $15 \%(1-60 \%)$ & $85 \%(50-100 \%)$ & $100 \%$ & $-\cdot$ \\
\hline $\begin{array}{l}\text { No. (\%) of positive cores: } \\
\text { SPBx } \\
\text { mpMRI-suspicious biopsy }\end{array}$ & $\begin{array}{l}7.5(26.7 \%) \\
2.5(62.6 \%)\end{array}$ & $\begin{array}{c}2.8(9.7 \%) \\
1.3(32.5 \%)\end{array}$ & $\begin{array}{c}12(42.8 \%) \\
3(75 \%)\end{array}$ & $\begin{array}{l}16(57.1 \% \\
3.2(80 \%)\end{array}$ & - \\
\hline $\begin{array}{l}\text { Median diameter (mm) of mpMRI suspicious area } \\
<10 \mathrm{~mm} \\
>10 \mathrm{~mm}\end{array}$ & $\begin{array}{c}12(5-25) \\
30 \\
22\end{array}$ & $\begin{array}{c}9(5-16) \\
23 \\
7\end{array}$ & $\begin{array}{c}16(9-32) \\
7 \\
11\end{array}$ & $\begin{array}{c}20(18-25) \\
0 \\
4\end{array}$ & $\begin{array}{c}6(5-13) \\
35 \\
5\end{array}$ \\
\hline
\end{tabular}

$35.3 \%$, respectively $(\mathrm{p}<0.05)$. Diagnostic accuracy, sensitivity, specificity, positive and negative predictive value of multiparametric mpMRI in diagnosing was $75.7 \%, 82.5 \%$, $71.8 \%, 78.9 \%, 87.9 \%$, respectively.

\section{Discussion}

Although TRUS imaging was enriched in recent years by the introduction of three-dimensional, computerized images and contrast media, which allow better characterization of intraparenchymal microvasculature (21), ultrasound accuracy is poor in performing targeted-biopsy $(22,23)$; therefore, SPBx still today remains the gold standard $(2,18)$ in case of repeat prostate biopsy. On the other hand, transrectal prostate biopsy, recently, has been associated with an increased risk of complications secondary to urinary tract infection and sepsis (1\% of the cases), with the necessity for hospital admission in $2 \%$ of the cases (24); conversely, transperineal prostate biopsy demonstrated a better accuracy in comparison with transrectal approach in the diagnosis of anterior zone $\mathrm{PCa}$ $(15,25)$, resetting the risk of sepsis $(26,27)$.

In the last years, mMRI has gained growing importance in PCa diagnosis and staging using mpMRI or meMRI (316); recently, 3 Tesla MRI has been suggested in the reevaluation of patients enrolled in active surveillance protocols (9), and is highly representative of the true GS $(12,14,15)$ and predictive of significant $\mathrm{PCa}(11,12$, 15). The estimated sensitivity and specificity for PCa detection by MRI varies between $57 \%$ and $100 \%$ vs $44 \%$ and $96 \%$, respectively (3); therefore, there is increasing interest in using MRI, especially in men with prior negative prostate biopsy and persistent suspicion of PCa. Multiparametric pMRI and eMRI have been introduced in clinical practice to detect suspicious areas which could be submitted to real-time MRI-guidance targeted-biopsy or translated into real-time MRI/TRUS imaging fusion to perform targeted biopsy $(4,7,11,16)$. Pinto et al. (4) showed a greater detection rate of cancer for each core using MRI imaging/ultrasound fusion-guided biopsy in comparison with standard 12-core transrectal biopsy (20.6\% vs $11.7 \%$, respectively). Franiel et al. (5) and Hambrock et al. (6) in patients with previous negative biopsy submitted to MRI-guided biopsy demonstrated a detection rate for PCa of $39 \%$ and 59\%, respectively; Kuru et al. (7), recently, evaluated the Prostate Imaging Reporting and Data System (PIRADS) in mpMRI based on single-core histology suggesting that PIRADS can be used as a decision-support system for targeting of suspicious lesions. Although eMRI had the best sensitivity and specificity in diagnosing and staging PCa, recently 3 Tesla pMRI demonstrated good accuracy in detecting areas suspicious for $\mathrm{PCa}$ and increasing diagnosis of cancer localized in the anterior zone of the gland $(5,15,25)$. The use of mpMRI provides, in daily practice, more advantages in comparison with meMRI; in fact, its use is widespread in many general hospitals, it is easy to perform and it does not generate discomfort to the patient. In addition, whole-body MRI has been suggested as a one-step procedure for staging men with high-grade PCa (28).

In our series, mpMRI detected 52 out 66 PCa; in detail, mpMRI in comparison with SPBx diagnosed 4 significant cancer of the anterior zone missing 12 cancers characterized by microfocal biopsy histological disease (18) at risk for insignificant $\mathrm{PCa}$ (22). In addition, a correlation between mpMRI suspicious lesion diameter vs PCa diagnosis and tumour grade was found

Some limitations and considerations of the present study deserve mention. Firstly, we do not know the true diagnostic accuracy of mpMRI in PCa diagnosis because the detection rate for cancer was compared with SPBx results. Secondly, we do not know if the false-positive rate (23.8\% of the cases) of mpMRI was secondary to false-negative SPBx results or was biased because an MRI imaging/ultrasound fusion-guided biopsy, theoretically more accurate, was not performed. In conclusion, in case of repeat biop- 
sy, mpMRI targeted biopsy improves diagnosis of anterior zone PCa missing cancers at risk for clinically insignificant disease; moreover, suspicious mpMRI lesions $>10 \mathrm{~mm}$ are highly predictive of aggressive PCa.

\section{References}

1. Pepe P, Aragona F: Prostate needle biopsy: 12 vs. 18 cores. Is it necessary? Urol Int. 2005; 74:19-22.

2. Chun FK, Epstein JI, Ficarra V, et al. Optimizing performance and interpretation of prostate biopsy: A critical analysis of the literature. Eur Urol 2010; 58:851-864.

3. Lawrentschuck N, Fleshner N. The role of magnetic resonance imaging in targeting prostate cancer in patients with previous negative biopsies elevated prostatic-specific antigen levels. BJU Int. 2009; 103:730-733.

4. Pinto PA, Chung PH, Rastinehad AR, et al. Magnetic resonance imaging/ultrasound fusion guided prostate biopsy improves cancer detection following transrectal ultrasound biopsy and correlates with multiparametric magnetic resonance imaging. J Urol. 2011; 186:1281-1285.

5. Franiel T, Stephan C, Erbersdobler A, et al. Areas suspicious for prostate cancer: MR-guided biopsy in patients with at least on US-guided biopsy with a negative finding. Multiparametric MR imaging for detection and biopsy planning. Radiology. 2011; 259:162-172.

6. Hambrock T, Somford DM, Hoeks C, et al. Magnetic resonance imaging-guided prostate biopsy in men with repeat negative biopsies and increased prostate-specific antigen. J Urol. 2010; 185:520-527.

7. Kuru TH, Roethke MC, Rieker P, et al. Histology core-specific evaluation of the European Society of Urogenital Radiology (ESUR) standardised scoring system of multiparametric magnetic resonance imaging (mpMRI) of the prostate. BJU Int. 2013; 112:1080-1087.

8. Rosenkrantz AB, Mussi Mendrinos S, Babb JS, Taneja SS: Prostate cancer foci detected on multiparametric resonance imaging are distinct from those not detected. J Urol. 2012; 187:2032-2038.

9. Quentin M, Biondin D, Klasen J, et al. Evaluation of a structured report of functional prostate magnetic resonance imaging in patients with suspicion for prostate cancer or under active surveillance. Urol Int. 2012; 89:25-29.

10. Hambrock T, Hoeks C, Hulsbergen-van de Kaa C, et al. Prospective assessment of prostate cancer aggressiveness using 3-T diffusion-weighted magnetic resonance image-guided biopsies versus systematic 10-core transrectal ultrasound prostate biopsy. Eur Urol. 2012; 61:177-184.

11. Turkbey B, Shah VP, Pang Y, et al. Is apparent diffusion coefficient associated with clinical risk scores for prostate cancers that are visible on 3-T MRI images? Radiology. 2011; 488-493.

12. Turkbey B, Pinto PA, Mani H, et al. Prostate cancer: Value of multiparametric MRI imaging at 3 T for detection-histopathologic correlation. Radiology. 2010; 255:89-94.

13. Arsov C, Quentin M, Rabenalt R, et al. Repeat transrectal ultrasound biopsies with additional targeted cores according to results of functional prostate MRI detects high-risk prostate cancer in patients with previous negative biopsy and increased PSA - a pilot study. Anticancer Res. 2012; 32:1087-1092.

14. Turkbey B and Choyke PL. Multiparametric MRI and prostate cancer diagnosis and risk stratification. Curr Opin Urol. 2012; 22:310-315.

15. Pepe P, Garufi A, Priolo G, et al. Prostate cancer detection at repeat biopsy biopsy: can pelvic phased-array multiparametric MRI replace saturation biopsy? Anticancer Res. 2013; 33:1195-1199.

16. Sonn GA, Natarajan S, Margolis DJ, et al. Targeted biopsy in the detection of prostate cancer using an office based magnetic resonance ultrasound fusion device. J Urol. 2013; 189:86-92.
17. Pepe P, Aragona F. Incidence of insignificant prostate cancer using free/total PSA: Results of a case-finding protocol on 14,453 patients. Prostate Cancer Prostatic Dis. 2010; 13:316-319.

18. Pepe P, Aragona F. Saturation prostate needle biopsy and prostate cancer detection at initial and repeat evaluation. Urology. 2007; 70:1131-1135.

19. Pepe P, Candiano G, Fraggetta F, et al. Is a single focus of low grade prostate cancer, diagnosed on saturation biopsy, predictive of clinically insignificant cancer? Urol Int. 2010; 84:440-444.

20. Epstein J, Walsh P, Carmichael M. Pathological and clinical findings to predict tumor extent of non palpable (stage T1c) prostate cancer. JAMA. 1994; 271:368-374.

21. Mitterberger M, Horninger W, Pelzer A, et al. A prospective randomized trial comparing contrast-enhanced targeted versus systematic ultrasound guided biopsies: Impact on prostate cancer detection. Prostate. 2007; 67:1537-1542.

22. Pepe P, Panella P, Patanè D, Aragona F. Does the adjunct of echographic contrast medium Levovist improve the detection rate of prostate cancer? Prostate Cancer Prostatic Dis. 2003; 6:159-162.

23. Pepe P, Candiano G, Pennisi M, Aragona F. Can Sonovue targeted biopsy replace extended or saturation biopsy in prostate cancer diagnosis? Our experience at primary and repeat biopsy. Arch It Urol Androl. 2010; 82:155-159.

24. Pinkhasov GI, Lin YK, Palmerola R, et al. Complications following prostate needle biopsy requiring hospital admission or emergency department visits - experience from 1000 consecutive cases. BJU Int. 2012; 110:369-374.

25. Komai Y, Numao N, Yoshida S, et al. High diagnostic ability of multiparametric magnetic resonance imaging to detect anterior prostate cancer missed by transrectal 12-core biopsy. J Urol. 2013; 190:867-873.

26. Pepe P, Aragona F: Morbidity following transperineal prostate biopsy in 3,000 patients submitted to 12 VS 18 VS more than 24 needle cores. Urology. 2013; 81:1142-1146.

27. Pepe P, Aragona F. Prostate biopsy: results and advantages of the transperineal approach-twenty-year experience of a single center. World J Urol. 2013 Jun 7. [Epub ahead of print].

28. Lecouvet FE, Mouedden JE, Collette L. Can whole-body magnetic resonance imaging with diffusion-weighted imaging replace $99 \mathrm{~m} \mathrm{Tc}$ bone scanning and computed tomography for single-step detection of metastases in patients with high risk prostate cancer? Eur Urol. 2012; 62:68-75

\section{Correspondence}

Pietro Pepe, MD (Corresponding Author) - piepepe@hotmail.com Giuseppe Dibenedetto, MD

Michele Pennisi, MD

Francesco Aragona, MD

Urology Unit, Cannizzaro Hospital, Via Messina 829, Catania, Italy

Antonio Garufi, MD

Giandomenico Priolo, MD

Imaging Department, Cannizzaro Hospital,

Via Messina 829, Catania, Italy

Michele Salemi, MD

Section of Endocrinology, Andrology and Internal Medicine, Department of Medical and Pediatric Sciences, University of Catania, Catania, Italy

Filippo Fraggetta, MD

Pathology Unit, Cannizzaro Hospital, Via Messina 829, Catania, Italy

Michele Barbera, MD

Urology Unit, Sciacca, Italy 